Tropical Journal of Pharmaceutical Research June 2015; 14 (6): 1103-1110

ISSN: $1596-5996$ (print); 1596-9827 (electronic)

(C) Pharmacotherapy Group, Faculty of Pharmacy, University of Benin, Benin City, 300001 Nigeria.

All rights reserved.

Available online at http://www.tjpr.org

Original Research Article

http://dx.doi.org/10.4314/tjpr.v14i6.24

\title{
Assessment of Knowledge, Interventional Practices for, and Impact of Malaria in Pregnancy among Parturient Women in a Nigerian Tertiary Healthcare Facility
}

\author{
Anthonia O Obieche ${ }^{1 *}$, Ehijie FO Enato ${ }^{1}$ and Adedapo BA Ande ${ }^{2}$ \\ ${ }^{1}$ Department of Clinical Pharmacy and Pharmacy Practice, Faculty of Pharmacy, ${ }^{2}$ Department of Obstetrics and Gynaecology, \\ College of Medical Sciences, University of Benin, Benin City, Nigeria
}

*For correspondence: Email: obumneke.obieche@uniben.edu

Received: 23 November 2014

Revised accepted: 19 May 2015

\begin{abstract}
Purpose: To assess knowledge of and intervention for malaria in pregnancy among immediate postpartum women, as well as the impact of malaria on some specific birth outcomes.

Methods: A cross-sectional study in which 358 immediate postpartum women were recruited in the labour and maternity wards of University Benin Teaching Hospital, Benin City, Nigeria. Information on demographics, knowledge of malaria, and utilization of malaria interventions were assessed. Immediately after expulsion of placentae, both maternal and placental blood samples were collected from a subset (236) of the study group for parasitaemia and haematocrit determination.

Results: The mean age of the women was $29.5 \pm 4.55$ years. Poor knowledge of adverse effects of malaria during pregnancy was observed. Using an arbitrary scale, low, average and high knowledge of malaria were recorded in $22.4,50.3$ and $27.3 \%$ of the respondents, respectively. Use of insecticidetreated net (ITN) was reported by 48.9, 19.1 and $14.3 \%$ of women with high, average and low knowledge of malaria respectively $(p=0.004)$ while there was no statistical difference in the use of sulphadoxine/pyrimethamine (IPTp-SP) among the different levels of knowledge of malaria as its practice was reported by 61,70 and $78.7 \%$ of women with high, average, and low knowledge of malaria, respectively, $(p=0.078)$. Incidence rates of parasitaemia of $4.0,1.6$ and $6.25 \%$ were obtained using peripheral microscopy, placental microscopy and peripheral rapid diagnostic test (RDT), respectively. Maternal infection was significantly associated with low birth weight $(p=0.020$, peripheral microscopy; $p=0.020$, placental microscopy) and maternal anaemia ( $p=0.009$, peripheral microscopy; $p=0.000$, peripheral RDT).

Conclusion: Knowledge gap still exists with regard to malaria and its interventions among mothers. Knowledge of malaria significantly influences the use of insecticide-treated bed nets. The negative impact of malaria infection during pregnancy is maternal anaemia and low birth weight infants.
\end{abstract}

Keywords: Insecticide-treated bed nets, Low birth weight, Malaria in pregnancy, Maternal anaemia.

Tropical Journal of Pharmaceutical Research is indexed by Science Citation Index (SciSearch), Scopus, International Pharmaceutical Abstract, Chemical Abstracts, Embase, Index Copernicus, EBSCO, African Index Medicus, JournalSeek, Journal Citation Reports/Science Edition, Directory of Open Access Journals (DOAJ), African Journal Online, Bioline International, Open-J-Gate and Pharmacy Abstracts

\section{INTRODUCTION}

Plasmodium falciparum malaria in pregnancy is a major public health problem in sub-Saharan Africa, posing serious health concern to pregnant women and the fetus. It is often asymptomatic and severe, as it often progresses undetected to complicated malaria [1]. The clinical consequences of the disease include maternal anaemia, low birth weight, preterm delivery, and by extension, increased perinatal and infant mortality $[2,3]$. Consequently, the protection of 
pregnant women living in malaria-endemic countries has become of particular interest to many international and national malaria control programmes. The World Health Organization recommends a three-prong approach to the control of malaria in pregnancy [4]. These are the practice of sleeping under insecticide-treated mosquito bed nets (ITNs) daily, intermittent preventive treatment in pregnancy using an effective anti-malarial drug e.g. sulphadoxinepyrimethamine (IPTp-SP), and effective management of clinical cases of malaria in pregnancy. Despite these recommendations however, there are reports indicating poor utilization of these interventions, particularly the use of ITNs and IPTp-SP $[5,6]$. The consequence of low utilization of aforementioned malaria control measures would be unresolved burden of malaria in pregnancy in endemic countries, including Nigeria. This study was undertaken in order to re-assess malaria situation during pregnancy in the light of recent attention by both local and national governments. Therefore, the objectives of this study were to assess knowledge of and intervention for malaria in pregnancy, as well as the impact of malaria on some specific birth outcomes among immediate postpartum women who delivered their babies in a tertiary healthcare facility in Nigeria.

\section{EXPERIMENTAL}

The study was a non-interventional and crosssectional study designed to assess the knowledge of, and interventional practices for malaria in pregnancy, as well as to determine the clinical outcomes in women diagnosed of malaria at parturition. The study was carried out between 1st April and 31st August 2011 at the University of Benin Teaching Hospital, Benin City, Edo State, Nigeria. The period of the study was during the high malaria transmission season in the area. This area had earlier been reported to experience monthly variations in delivery rates with peak delivery rates in April - May and October and lowest rates in July - August and December [7]. Women who delivered their babies (dead or alive) were approached immediately after delivery and given information regarding research objectives and were assured of confidentiality of personal information. Only those women who gave informed written or verbal consent and satisfied the inclusion criteria were enrolled in the study. The study protocol was reviewed and approved by the Ethical Review Committee of the University of Benin Teaching Hospital (Protocol number: ADM / E. 22A / VOL. VII / 392).
A total of 358 women were recruited consecutively during week-days into the knowledge and practice (KP) study. Excluded from the study were health care personnel, mentally unstable mothers and women who had history of allergy to sulphur-containing drugs. The women were interviewed with the aid of a pre-tested structured questionnaire (administered within 24 hours of delivery) which assessed the women's knowledge of malaria in pregnancy and practices of malaria control measures. Relevant information on socio-demographic characteristics and obstetric history of the participants were extracted from the women's medical case files.

The assessment of knowledge of malaria consisted of questions on four areas: transmission of malaria, knowledge of risk, preventive measures (the two recommended measures) and consequences of disease during pregnancy (any 3 consequences); appropriate responses to the questions corresponded to scores 1, 1, 2 and 3 respectively, as described elsewhere [8]. The range of possible scores was $0-7$. An arbitrary scale was used in categorizing the respondents. A mid-point of 3-4 was termed average knowledge. Below the mid-point was classified as "low knowledge score" while above was classified as "high knowledge score". Laboratory investigations for malaria at parturition consisted of peripheral microscopy, peripheral rapid diagnostic test (RDT) for parasite histidine rich protein 2 (HRP-2), and placental microscopy. Both maternal peripheral and placental (incision method) blood samples were collected immediately after delivery from a subset of the study participants $(n=224)$. Only study participants who gave consent for their blood samples to be collected when approached during labour, and who delivered while the investigator was present at the labour wards were sampled for laboratory investigation for malaria. This was important to avoid coagulation of the placental blood. Thick and thin blood smears of finger-prick peripheral blood sample from each woman were prepared on a slide for microscopic examination. Same procedure was done using the placental blood samples. Thereafter, the blood smears were air-dried and subsequently stained with $10 \%$ solution of freshly prepared Giemsa stain at $\mathrm{pH} 7.2$ for about $15 \mathrm{~min}$. The stained films were read by independent two microscopists. Detection of parasite HRP-2 in the maternal peripheral blood samples was done using a commercially available kit (SD Bioline Malaria Ag P. f test, Korea), according to the manufacturer's instruction. Also, samples from the maternal finger-prick blood were used for haematocrit determination. Excluded from the study were 
women who received blood and or blood products later than one week prior to their delivery, women who were diabetic during the pregnancy, or had physical trauma, or had multiple pregnancy or are known homozygous sickle cell disease patients for obvious reasons. Pregnancy outcomes that were assessed were low birth weight (LBW), defined as a birth weight $<2500 \mathrm{~g}$; preterm delivery, defined as gestational period < 37 weeks, and maternal anaemia, defined as packed cell volume < $30 \%$. Gestational period was assessed using the woman's last menstrual period (LMP) and validated with the first trimester ultrasound.

\section{Statistical analysis}

The data were entered in Microsoft Excel data sheets, cross checked and transferred, and analyzed using SPSS for Windows version 16.0 (SPSS, Atlanta, GA, USA). Descriptive statistics were carried out for continuous variables (mean $\pm \mathrm{SD}$ ), while categorical variables were treated as frequencies and percentages. Chi-squared test was used to compare proportions of categorical variables and to determine the statistical significance between the variables, while between groups comparisons were evaluated by Student's unpaired t-test and oneway ANOVA.

\section{RESULTS}

\section{Demographics of study participants}

Out of the 358 postpartum women who were administered questionnaires, complete data were obtained for 344 women giving a response rate of $96.1 \%$. The ages of the respondents ranged from $18-45$ years with a mean age of $29.5 \pm$ 4.55 year. Women within $26-35$ years age groups $(73.2 \%)$ constituted significant proportion of the respondents. The study group was predominantly made up of monogamously married women (94.2\%). More than two-fifths of the study group were primiparous women. All the women were formally educated. The characteristics of the participants are shown in Table 1. The mean duration of pregnancy and mean weight of the babies were $274.40 \pm 12.64$ days and $3.09 \pm 0.52 \mathrm{~kg}$, respectively.

\section{Knowledge of malaria in pregnancy}

A mean value of knowledge of malaria score of $3.53 \pm 1.397$ was obtained. The proportions of women with low, average and high knowledge were $22.4,50.3$ and $27.3 \%$, respectively.
On assessment of the cause of malaria, about 80 $\%$ (276) of the respondents attributed the cause of malaria to bites from infected mosquito only, while about $14 \%(48)$ of the respondents agreed that malaria could arise from bites from infected mosquito as well as other causes, such as, dirty environment, eating unclean food and drinking contaminated water, excessive exposure to sunlight, and excessive stress.

Table 1: Characteristics of participants $(n=344)$

\begin{tabular}{lcc}
\hline Variable & No. & $\%$ \\
\hline Age (years) & 8 & \\
$15-20$ & 47 & 2.3 \\
$21-25$ & 159 & 13.7 \\
$26-30$ & 93 & 46.2 \\
$31-35$ & 32 & 27.0 \\
$36-40$ & 5 & 9.3 \\
$>40$ & & 1.5 \\
Marital status & & \\
$\quad$ Married & 329 & 95.6 \\
Single & 13 & 3.8 \\
Unmarried but living with & 2 & 0.6 \\
partner & & \\
Parity & & \\
$\quad$ Primiparae & 154 & 44.8 \\
$\quad$ Secundiparae & 84 & 24.4 \\
$\quad$ Multiparae ( $\geq 3$ deliveries) & 106 & 30.8 \\
Educational level & & \\
$\quad$ Primary education & 17 & 5.0 \\
$\quad$ Secondary education & 127 & 36.9 \\
$\quad$ Tertiary education & 200 & 58.1 \\
Occupation & & \\
$\quad$ Unemployed & 87 & 25.3 \\
$\quad$ Petty trader & 112 & 32.6 \\
Classic business woman & 53 & 15.4 \\
$\quad$ Civil servant & 86 & 25.0 \\
Public servant & 6 & 1.7 \\
\hline & & \\
\hline & &
\end{tabular}

An appreciable proportion of the women (88.7 \%, $305 / 344$ ) agreed that pregnancy increases the risk of malaria; while the remaining $11.3 \%$ (39/344) had a contrary opinion (25 women agreed that pregnancy does not increase risk of malaria, while 14 women agreed that the risk is equal in both pregnant and non-pregnant women). However, $47.2 \%(144 / 305)$ of the women who agreed that pregnancy increases the risk of malaria had no reason for their response. The reasons given by other women for increased susceptibility to malaria during pregnancy are decreased maternal immunity (22.4\%), weak body and inadequate care of oneself $(19.5 \%)$, physiological changes due to foetal presence (7.6\%), anorexia and vomiting (2.2\%), and bad blood and water in the maternal circulation (0.9 $\%)$.

The lowest knowledge scores related to the impact of maternal malaria on the health of the foetus; about $50 \%$ of the respondents failed to 
recognize any potential consequence of maternal malaria on the foetus. Only $34.6 \%$ (119), $12.2 \%$ (42) and $2.9 \%$ (10) of the women mentioned one, two and three adverse effects of malaria in pregnancy (MiP), respectively. Of all the consequences of MiP that were mentioned, maternal anaemia was most cited $(49.1 \%, \mathrm{n}=$ 84/171). Other consequences of MiP that were mentioned by the respondents were jaundice in new born babies (31.6\%), pregnancy loss $(25.1$ $\%)$, malaria in baby (17\%), low birth weight (8.2 $\%)$, newborn death $(4.1 \%)$ and intrauterine feotal death $(1.8 \%)$.

Of the 344 respondents, only 109 (31.7\%) women knew that both intake of sulphadoxine/pyrimethamine-based intermittent preventive therapy (IPTp-SP) and the use of insecticide-treated bed nets (ITNs) are the two best anti-malarial control measures during pregnancy. A significant proportion of the respondents $(77.6 \%)$ were aware of ITNs than IPTp-SP $(41.3 \%)$. Other interventions that were mentioned by the respondents for effective control of MiP include: indoor insecticide spraying (16\%; $n=55)$, healthy eating and drinking habits (16.3\%; $n=56)$, environmental sanitation (9.0\%; $n=31)$, and use of local herbs $(2.9 \% ; n=10)$. A greater proportion of the respondents $(37.2 \% ; n=128)$ believed that netted doors and windows are effective preventive measures against pregnancy.

\section{Reported anti-malarial interventions during pregnancy}

The study participants used eight different antivector measures - (insecticide treated bed net, untreated bed net, indoor insecticide spraying, door and window nets, repellant creams and soap, insecticide coils, local herbs and protective wears). Of the 8 anti-vector measures that were identified, door and window nets had the highest rate of use $(90.4 \%)$. The respondents' opinion of best anti-malarial measure for malaria in pregnancy was at variance with what they practiced during pregnancy (Table 2).

Despite the high level of awareness of insecticide-treated bed nets (ITNs) among the respondents, the utilization rate among the respondents was poor $(26.2 \%$, OR: 5.432 ; Cl: 2.27 - 12.99). Intermittent preventive treatment using sulphadoxine/pyrimethamine (IPTp-SP) was utilized by a proportion of women higher than the proportion that knew about it $(70.3 \%$, OR: 1.269; Cl: 0.79 - 2.04). The IPTp-SP was reportedly not taken at all by $29.7 \%$ (102) of the women. Notwithstanding the foregoing, appropriate use of these interventions was further recorded in a smaller proportion of the women. Only $6.7 \%$ (23) of the interviewed women slept under ITNs daily, while $32 \%$ (110) of the women utilized more than one dose of IPTp-SP. Only $10(2.9 \%)$ women utilized both daily ITN and $\geq$ two doses of IPTp-SP. The mean gestational period (weeks) of intake of first and second doses of IPTp-SP were 24 and 32, respectively.

Table 2: Respondents' opinion of best anti-malarial measures versus actual practice during pregnancy ( $\mathrm{n}$ $=344$ )

\begin{tabular}{|c|c|c|}
\hline Intervention & $\begin{array}{c}\text { Opinion as } \\
\text { an } \\
\text { effective } \\
\text { measure } \\
\text { N (\%) }\end{array}$ & $\begin{array}{c}\text { Actual } \\
\text { practice } \\
\text { during } \\
\text { pregnancy } \\
\mathbf{N}\left(\%{ }^{*}\right)\end{array}$ \\
\hline ITNs & $267(77.6)$ & $90(26.2)$ \\
\hline IPTp - SP & $142(41.3)$ & $242(70.3)$ \\
\hline ITNs + IPTp-SP & 109 (31.7) & $22(6.4)$ \\
\hline Door and window nets & $128(37.2)$ & $311(90.4)$ \\
\hline Indoor insecticide spraying & $55(16)$ & $282(82)$ \\
\hline Local herbs & $10(2.9)$ & $10(2.9)$ \\
\hline Healthy eating/drinking habits & 56 (16.2) & - \\
\hline Environmental sanitation & $31(9)$ & - \\
\hline Untreated bed net & - & $11(3.2)$ \\
\hline Insecticide coil & - & $3(0.9)$ \\
\hline Repellant cream and soap & - & $15(4.4)$ \\
\hline Protective wears & - & $107(31.1)$ \\
\hline Non-SP prophylaxis & & $35(10.2)$ \\
\hline \multicolumn{3}{|c|}{$\begin{array}{l}\text { Percentage is more than } 100 \% \text { because some } \\
\text { respondents used more than one anti-malarial } \\
\text { intervention. } N=\text { number of women; ITN = insecticide- } \\
\text { treated bed nets, IPTp-SP = intermittent preventive } \\
\text { treatment in pregnancy using sulphadoxine- } \\
\text { pyrimethamine }\end{array}$} \\
\hline
\end{tabular}

Reasons given by the women for non-use of ITNs during the immediate past pregnancy were: lack of awareness (5.9\%), believed that door and window nets were sufficient control measures $(17.7 \%)$, inaccessibility (12.6\%), disliked by the husband (8.7\%), meant for children $(12.6 \%)$, lack of fund $(17.7 \%)$, causes discomfort and sweating (11.8\%), unnecessary because they believed they have immunity $(3.1$ $\%)$, did not have mosquitoes in the house (1.2 $\%)$, and difficulty to properly fix the net at home $(8.7 \%)$. Among the IPTp-SP defaulters were women who believed that the drug is not safe in pregnancy $(n=27)$, women who said that the intake of the drug is unnecessary since they had no malaria symptoms at the time they were given the medication $(n=15)$ and those who had late booking for antenatal care services, or presented at term or in labour $(n=60)$. 
Clinical outcomes among users of different anti-malarial interventions during pregnancy

The practice of intake of at least 2 doses of IPTp$\mathrm{SP}$ and sleeping in ITNs daily during pregnancy was associated with good maternal and neonatal outcomes (Table 3). However, women that used other anti-malarial interventions had varying degrees of poor pregnancy outcomes. The poorest clinical outcomes - highest frequency of incidence of clinically diagnosed malaria during pregnancy, number of babies with LBW and preterm delivery were observed among those women that used untreated door and window nets only.

\section{Pregnancy outcomes and interventional practices associated with knowledge of malaria}

Between the three levels of knowledge of malaria, there was no significant difference in the mean birth weight and mean duration of pregnancy in days (Table 4). The mean maternal PCV was significantly high in mothers with high KoM $(25.22 \pm 16.795, p=0.003)$. Use of ITN was reported by $48.9 \%, 19.1 \%$ and $14.3 \%$ of women with high, average and low knowledge of malaria respectively $(p=0.004)$. On the other hand, there was no statistical difference in the use of IPTp-SP among the different levels of knowledge of malaria as its practice was reported by $61 \%, 70 \%$ and $78.7 \%$ of women with high, average, and low knowledge of malaria, respectively, $(p=0.078)$.

\section{Incidence of, and pregnancy outcomes associated with malaria at parturition}

By microscopy, $P$. falciparum malaria-positive results were obtained in 9/224 (4.0\%) and 4/224 $(1.6 \%)$ peripheral and placental blood samples, respectively. Peripheral RDT gave malariapositive results for 14/224 $(6.25 \%)$ blood samples. Only three peripheral blood samples were positive by both microscopy and RDT. The peripheral blood films of the 4 women with placental infection all showed negative results by both microscopy and RDT. Overall, 24 women were malaria-positive. This gave a rate of $P$. falciparum malaria at parturition of $10.7 \%$.

All women who tested positive for malaria had average or high knowledge of malaria in pregnancy. However, they had poor adherence to the recommended preventive measures for malaria in pregnancy - sleeping under ITNs daily and intake of at least two doses of IPTp-SP. Only

Table 3: Outcomes observed among users of different anti-malarial interventions during pregnancy

\begin{tabular}{|c|c|c|c|c|}
\hline Anti-malarial intervention & $\begin{array}{c}\text { No. of babies } \\
\text { with LBW } \\
\text { N (\%) }\end{array}$ & $\begin{array}{c}\text { Preterm } \\
\text { delivery, } \quad \mathrm{N} \\
(\%)\end{array}$ & $\begin{array}{l}\text { MiP cases } \\
\quad \text { N (\%) }\end{array}$ & $\begin{array}{c}\text { Maternal } \\
\text { anaemia } \\
\mathrm{N}(\%)\end{array}$ \\
\hline Daily ITN use only $(n=8)$ & $2(25)$ & $2(25)$ & $5(62.5)$ & 1(12.5) \\
\hline $\begin{array}{l}\text { At least } 2 \text { doses of IPTp-SP only } \\
(n=16)\end{array}$ & $0(0)$ & $1(6.3)$ & $6(37.5)$ & $2(12.5)$ \\
\hline $\begin{array}{l}\text { Regular indoor insecticide spraying } \\
\text { only }(n=11)\end{array}$ & $1(9.1)$ & $0(0)$ & $9(81.8)$ & $3(27.3)$ \\
\hline $\begin{array}{l}\text { Untreated door and window nets } \\
\text { only }(n=12)\end{array}$ & $5(41.7)$ & $4(33.3)$ & $5(41.7)$ & $3(25)$ \\
\hline $\begin{array}{l}\text { Daily ITN use }+ \text { at least } 2 \text { doses of } \\
\text { IPTp - SP }(n=10)\end{array}$ & $0(0)$ & $0(0)$ & $1(10.0)$ & $0(0)$ \\
\hline
\end{tabular}

MiP = malaria in pregnancy; $L B W=$ low birth weight; ITN = insecticide-treated bed nets, IPTp-SP = intermittent preventive treatment in pregnancy using sulphadoxine-pyrimethamine; $N=$ number of women who experienced the poor outcome

Table 4: Comparison of pregnancy outcomes between the three levels of knowledge of malaria

\begin{tabular}{lcccc}
\hline & \multicolumn{2}{c}{ Level of knowledge of malaria } \\
\hline Pregnancy outcome & Low knowledge & $\begin{array}{c}\text { Average } \\
\text { knowledge }\end{array}$ & High knowledge & P-value \\
\hline Mean birth weight $(\mathrm{kg})$ & $2.98 \pm .551$ & $3.1046 \pm 0.534$ & $2.9773 \pm .551$ & 0.165 \\
Mean duration of pregnancy (days) & $274.12 \pm 13.768$ & $274.92 \pm 11.883$ & $273.68 \pm 13.132$ & 0.554 \\
Mean PCV (\%) of respondents ${ }^{\mathrm{a}}$ & $22.27 \pm 17.039$ & $21.80 \pm 17.680$ & $25.22 \pm 16.795$ & $\mathbf{0 . 0 0 3}$ \\
\hline
\end{tabular}

${ }^{a}$ Number of respondents $=224$; values are presented as mean $\pm S D$; statistical analysis is based on one-way ANOVA; $p<0.05$ was considered statistically significant; $P C V=$ parked cell volume 
Table 5: Comparison of pregnancy outcomes among malaria-positive and malaria-negative mothers identified by peripheral microscopy, peripheral RDT and placental microscopy

\begin{tabular}{|c|c|c|c|c|c|c|c|c|}
\hline \multirow[t]{2}{*}{ Test } & \multicolumn{3}{|c|}{ Mean birth weight (Kg) } & \multicolumn{2}{|c|}{ Mean maternal PCV (\%) } & \multicolumn{2}{|r|}{$\begin{array}{l}\text { Mean duration of } \\
\text { pregnancy (days) }\end{array}$} & \multirow[b]{2}{*}{ P-value } \\
\hline & $\begin{array}{l}\text { Malaria } \\
\text { positive }\end{array}$ & $\begin{array}{l}\text { Malaria } \\
\text { negative }\end{array}$ & $P$ value & $\begin{array}{l}\text { Malaria } \\
\text { positive }\end{array}$ & $\begin{array}{l}\text { Malaria } \\
\text { negative }\end{array}$ & $P$ value & $\begin{array}{ll}\text { Malaria } & \text { Malaria } \\
\text { positive } & \text { negative }\end{array}$ & \\
\hline $\begin{array}{l}\text { Peripheral } \\
\text { microscopy }\end{array}$ & $2.82 \pm 0.45$ & $3.12 \pm 0.39$ & 0.020 & $30.33 \pm 3.35$ & $35.22 \pm 5.50$ & 0.009 & $272.11 \pm 11.91276 .22 \pm 9.83$ & 0.224 \\
\hline $\begin{array}{l}\text { Peripheral } \\
\text { RDT }\end{array}$ & $2.92 \pm 0.37$ & $3.13 \pm 0.40$ & 0.088 & $28.36 \pm 5.41$ & $35.37 \pm 5.32$ & 0.000 & $272.82 \pm 10.72276 .22 \pm 9.88$ & 0.268 \\
\hline $\begin{array}{l}\text { Placental } \\
\text { microscopy }\end{array}$ & $2.44 \pm 0.28$ & $3.1 \pm 0.39$ & 0.000 & $32.00 \pm 3.16$ & $35.14 \pm 0.36$ & 0.256 & $280.25 \pm 4.99275 .98 \pm 9.98$ & 0.395 \\
\hline
\end{tabular}

Data are mean $\pm S D$; statistical analysis is based on Student's t-test; $p<0.05$ was considered statistically significant; $P C V=$ packed cell volume; $R D T=$ rapid diagnostic test

5 women used ITNs while 9 women took only one dose of SP. None of the women took second dose of IPTp-SP. Almost all the women (23/24) mentioned that they had untreated nets fixed on the doors and windows at home.

Overall, the birth weight of the babies born to the women that were assessed $(n=224)$ ranged from 2 to $4.25 \mathrm{~kg}(3.14 \pm 0.37 \mathrm{~kg})$. The maternal PCV and duration of pregnancy ranged from 17 to $52 \%(22.84 \pm 17.31 \%)$ and 205 to 295 (274 \pm 12.64) days, respectively.

Eight women out of the 9 women who had malaria on peripheral blood examination delivered live babies whose birth weights ranged from $2.25-3.35 \mathrm{~kg}$ (mean weight \pm standard deviation). The remaining mother had an intrauterine foetal death of a $3.6 \mathrm{~kg}$ baby. Five women out of the nine women with malaria detectable microscopically in peripheral blood had $\mathrm{PCV}<30 \%$. Only 1 woman out of the 14 women with RDT-positive results had a baby with LBW, while 9 women out of them had maternal $\mathrm{PCV}<30 \%$. Two women out of the 3 women who tested positive by both peripheral microscopy and RDT had babies with LBW. The third woman had an intrauterine foetal death. Only 1 out of the 4 women with placental malaria had low maternal PCV of $29 \%$. However, LBW was recorded in three women. Table 5 shows that maternal infection was significantly associated with low birth weight, LBW $(p=0.020$, peripheral microscopy; $p=0.020$, placental microscopy), and maternal anaemia ( $p=0.009$, peripheral microscopy; $p=0.000$, peripheral RDT). Although maternal infection was not significantly associated with preterm delivery, yet the mean duration of pregnancy (days) was lower among the malaria-positive mothers confirmed by peripheral microscopy and RDT than in malaria-negative mothers.

\section{DISCUSSION}

Malaria remains one of the parasitic diseases of immense public health burden particularly in subSaharan Africa. This study reveals the existence of gaps in the knowledge of malaria in pregnancy, low rates of utilization of the recommended malaria preventive measures during pregnancy (below global targets) as well as poor maternal and neonatal outcomes associated with malaria in pregnancy.

The finding of women's poor knowledge of consequences of maternal malaria on the foetus despite having average or high general knowledge of malaria in pregnancy suggests need for adequate malaria education among women. Similar studies had reported low knowledge of foetal health impact of malaria in pregnancy among pregnant women [8,9]. Furthermore, a higher number of respondents were aware of ITN than IPTp-SP as preventive strategy for malaria in pregnancy. A comparable result was obtained in a similar study carried out among pregnant women presenting for antenatal care in Uyo, Nigeria [5]. Despite the high level of awareness of insecticide-treated bed nets (ITNs) among the respondents, the utilization rate was poorer than that of IPTp-SP. Thus, the popularly accepted fact that poor knowledge or awareness of anti-malarial interventions significantly accounts for the low utilization rates of these measures is contentious. Though a majority of the women used IPTp-SP, the knowledge of the rationale for the use of the drug during pregnancy in these women was low. A factor noted to have contributed to the high utilization rate of IPTp-SP at the study site was the routine prescription and dispensing of sulphadoxinepyrimethamine to the pregnant women during their antenatal clinic visits. Although a large proportion of the women utilized first dose of IPTp-SP at some point during the pregnancy, less than half of them took subsequent doses. Failure of the women to return at subsequent 
scheduled antenatal visits, resulting in incomplete intake of IPTp-SP doses might have been the contributing factor. It was also observed that intake of IPTp-SP doses, particularly when the second and or third dose was taken, occurred rather late into the pregnancy. This might have contributed to the low incidence rate of malaria at parturition. However, the delayed use of IPTp-SP during pregnancy may not adequately protect the growing foetus against malaria during the time of maximum foetal growth. Besides the ITNs and IPTp-SP, women used other anti-malarial control measures during the immediate past pregnancy which did not confer effective protection against adverse effects of malaria. None of the women who utilized both daily ITN and at least 2 doses of IPTp-SP had any form of poor maternal or neonatal outcome. These findings further support the benefits of the two interventional measures against malaria in pregnancy.

The rate of peripheral malaria obtained in this study was comparable to the rate reported in a recent Nigerian study [10]. However, varying rates of peripheral parasitaemia ranging from 7.5 $\%-60 \%$ had been reported in previous studies in different parts of Nigeria [11-13]. In addition, such wide variations had been observed in recent studies from other African countries, such as Kenya (ranging from $9.4 \%$ to $20.1 \%$ ) $[14,15]$; and Cameroun (ranging from $15.9 \%$ to $32.8 \%$ ) $[16,17]$. It is worthy of note that neither microscopy of peripheral blood nor HRP2 detection test in the peripheral blood could detect any of the $P$. falciparum infections confirmed by placental microscopy. The possibility of existence of placental parasitemia in the absence of peripheral blood parasitemia, and even the persistence of parasites in the placenta after initiation of anti-malarial treatment had been documented in previous report (1997) [18]. In this study, maternal malaria identified by peripheral microscopy and placental infection were associated with low birth weight. This result is consistent with the findings from previous studies though, Singer et al (2004) reported no association between peripheral parasitaemia and prevalence of low birth weight $[19,20]$. Positive peripheral RDT, as well as, peripheral microscopy were found to be significantly associated with reduced maternal packed cell volume (PCV). The result was similar to those of previous studies $[21,22]$. In the present study, we did not find association between maternal malaria and preterm delivery, which is similar to a previous report by Mokuolu et al [21]. However, some other studies had reported existence of such an association between maternal malaria and preterm delivery [23].

\section{Limitations of the study}

This study has limitations. The first limitation is recall bias that might be present during the interview as some women might not have accurately recalled the anti-malarial control measures they used during pregnancy. The second limitation is the small sample size that was used for the parasitological study.

\section{CONCLUSION}

Knowledge of malaria significantly influences the use of insecticide treated bed nets, but not intermittent preventive treatment by pregnant women. Considering the health burden of malaria during pregnancy, we recommend effective health educational programmes for proper public enlightenment on control of the disease. There may be need to review current strategies of educating the community about malaria control and its impacts.

\section{ACKNOWLEDGEMENT}

The authors thank the management and staff of the labour and maternity wards of the University of Benin Teaching Hospital, especially the nurses, who were of great assistance during data collection. All the women who participated in the study are gratefully acknowledged for their cooperation.

\section{REFERENCES}

1. Desowitz RS, Alpers MP. Placental Plasmodium falciparum parasitaemia in East Sepik (Papua New Guinea) women of different parity: the apparent absence of acute effects on mother and foetus. Ann Trop Med Parasitol 1992; 86 (2): 95-102.

2. Steketee RW, Nahlen BL, Parise ME, Menendez C. The burden of malaria in pregnancy in malaria-endemic areas. Am J Trop Med Hyg 2001; 64(suppl): 28-35.

3. Guyatt HL, Snow RW. The epidemiology and burden of plasmodium falciparum-related anaemia among pregnant women in sub-Saharan Africa. Am J Trop Med Hyg 2001; 64(suppl): 36-44.

4. WHO: Framework for developing, implementing and updating national antimalarial drug policy: A guide for country malaria control programmes. WHO Regional Office for Africa, Brazzaville. AFR/MAL/03.02 World Health Organization, 2003.

5. Abasiattai AM, Etukumana EA, Umoiyoho AJ. Awareness and Practice of malaria prevention strategies among pregnant women in Uyo, South South Nigeria. Internet J Gynecol Obstet 2009; 11(1).

6. Tongo OO, Orimadegun AE, Akinyinka OO. Utilisation of malaria preventive measures during pregnancy and 
birth outcomes in Ibadan, Nigeria. Pregnancy and Childbirth. 2011; 11: 60-67.

7. Enabudoso EJ, Okpighe AC, Gharoro EP, Okpere EE. Delivery rate in Benin City, Nigeria: Are there seasonal variations? Nig J Clin Pract 2011; 14 (2): 129-131.

8. Nganda RY, Drakeley C, Reyburn H, Marchant $T$. Knowledge of malaria influences the use of insecticide treated nets but not intermittent presumptive treatment by pregnant women in Tanzania. Malar J 2004; 3: 42-49.

9. Enato EFO, Okhamafe AO, Okpere EE. A survey of knowledge, attitude and practice of malaria management among pregnant women from two health care facilities in Nigeria. Acta Obstet et Gynecol Scand 2007; 86: 33-36.

10. Agomo CO, Oyibo WA, Anorlu RI, Agomo PU. Prevalence of malaria in pregnant women in Lagos, South-West Nigeria. Korean J Parasitol 2009; 47 (2): 179-183.

11. Okwa OO. The status of malaria among pregnant women: a study in Lagos, Nigeria. Afr J Reprod Health 2003; 7: 77-83.

12. VanderJagt TA, Ikeh El, Ujah IO, Belmonte J, Glew RH, VanderJagt DJ. Comparison of the OptiMAL rapid test and microscopy for detection of malaria in pregnant women in Nigeria. Trop Med Int Health 2005; 10: 39-41.

13. Enato EFO, Okhamafe AO, Okpere EE, Oseji FI. Prevalence of malaria during pregnancy and antimalarial intervention in an urban secondary health care facility in Southern Nigeria. Med Princ Pract 2007; 16: 240-243.

14. Malhotra I, Dent A, Mungai P, Muchiri E, King CL. Realtime quantitative PCR for determining the burden of Plasmodium falciparum parasites during pregnancy and infancy. J Clin Microbiol 2005; 43(8): 3630-3635.

15. van Eijk AM, Ayisi JG, ter Kuile FO, Misore AO, Otieno $J A$, Rosen DH, Kager PA, Steketee RW, Nahlen BL. Risk factors for malaria in pregnancy in an urban and peri-urban population in western Kenya. Trans $R$ Soc Trop Med Hyg 2002; 96: 586-592.

16. Tako EA, Zhou A, Lohoue J, Leke R, Taylor DW, Leke $R F$. Risk factors for placental malaria and its effect on pregnancy outcome in Yaoundé, Cameroon. Am J Trop Med Hyg 2005; 72: 236-242.

17. Akum AE, Kuoh AJ, Minang JT, Achimbom BM, Ahmadou MJ, Troye-Blomberg M. The effect of maternal, umbilical cord and placental malaria parasitaemia on the birth weight of newborns from South-western Cameroon. Acta Paediatr 2005; 94(7): 917-923.

18. Sartelet H, Milko-Sartelet I, Garraud O, Picot S. Plasmodium falciparum persist in the placenta after three days' treatment with quinine. Trans $R$ Soc Trop Med Hyg 1997; 91: 431.

19. Singer LM, Newman RD, Diarra A, Moran AC, Huber CS, Stennies G, Sirima SB, Konate A, Yameogo $M$, Sawadogo R, Barnwell JW, Parise ME. Evaluation of a malaria rapid diagnostic test for assessing the burden of malaria during pregnancy. Am J Trop Med Hyg 2004; 70(5): 481-485.

20. Wort UU, Warsame M, Brabin B. Birth outcomes in adolescent pregnancy in an area with intense malaria transmission in Tanzania. Acta Obstet Gynecol Scand 2006; 85(8): 949-954.

21. Mokuolu OA, Falade CO, Orogade AA, Okafor $H U$, Adedoyin OT, Oguonu TA, Dada-Adegbola HO, Oguntayo OA, Ernest SK, Hamer DH, Callahan MV. Malaria at Parturition in Nigeria: Current status and delivery outcome. Infect Dis in Obstet Gynecol 2009; doi:10.1155/2009/473971.

22. Egwunyenga OA, Ajayi JA, Duhlinska-Popova DD. Malaria in pregnancy in Nigerians: seasonality and relationship to splenomegaly and anaemia. Indian $\mathrm{J}$ Malariol 1997; 34(1): 17-24.

23. N'Dao CT, N'Diaye JL, Gaye A, Le Hesran JY. Placental malaria and pregnancy outcome in a peri urban area in Senegal. Rev Epidemiol Sante Publique 2006; 54(2): 149-156. 